\title{
Spin dynamics of isoelectronic bound excitons in $\mathrm{ZnO}$
}

Shula Chen, Weimin Chen and Irina Buyanova

\section{Linköping University Post Print}

\section{Tweet}

N.B.: When citing this work, cite the original article.

Original Publication:

Shula Chen, Weimin Chen and Irina Buyanova, Spin dynamics of isoelectronic bound excitons in ZnO, 2014, Physical Review B. Condensed Matter and Materials Physics, (89), 23, 235202.

http://dx.doi.org/10.1103/PhysRevB.89.235202

Copyright: American Physical Society http://www.aps.org/

Postprint available at: Linköping University Electronic Press

http://urn.kb.se/resolve?urn=urn:nbn:se:liu:diva-109184 


\title{
Spin dynamics of isoelectronic bound excitons in $\mathrm{ZnO}$
}

\author{
S. L. Chen, W. M. Chen, and I. A. Buyanova* \\ Department of Physics, Chemistry and Biology, Linköping University, 58183 Linköping. Sweden
}

(Received 28 March 2014; revised manuscript received 28 May 2014; published 10 June 2014)

\begin{abstract}
Time-resolved optical spin orientation is employed to study spin dynamics of $I^{*}$ and $I_{1}^{*}$ excitons bound to isoelectronic centers in bulk $\mathrm{ZnO}$. It is found that spin orientation at the exciton ground state can be generated using resonant excitation via a higher lying exciton state located at about $4 \mathrm{meV}$ from the ground state. Based on the performed rate equation analysis of the measured spin dynamics, characteristic times of subsequent hole, electron, and direct exciton spin flips in the exciton ground state are determined as being $\tau_{h}^{s}=0.4 \mathrm{~ns}, \tau_{e}^{s} \geqslant$ $15 \mathrm{~ns}$, and $\tau_{e h}^{s} \geqslant 15 \mathrm{~ns}$, respectively. This relatively slow spin relaxation of the isoelectronic bound excitons is attributed to combined effects of (i) weak $e$ - $h$ exchange interaction, (ii) restriction of the exciton movement due to its binding at the isoelectronic center, and (iii) suppressed spin-orbit coupling for the tightly bound hole.
\end{abstract}

DOI: 10.1103/PhysRevB.89.235202

PACS number(s): 72.25.Fe, 78.55.Et

\section{INTRODUCTION}

There is considerable current interest in $\mathrm{ZnO}$, a II-VI semiconductor which has a wide bandgap of $3.37 \mathrm{eV}$ and a large exciton binding energy of $60 \mathrm{meV}$ that are considered as key properties for applications in ultraviolet (UV) optoelectronic and white light emitting devices (for a review, see e.g. Refs. [1$3]$ ). Besides the prominent optical performance, $\mathrm{ZnO}$ is also a promising material for spin-functional electronic devices and spin-based quantum computation. First of all, when alloyed with transition metals, it has been shown to exhibit ferromagnetic properties at and above room temperature (RT) [4-6] desirable for generation of spin-polarized free carriers. Secondly, $\mathrm{ZnO}$ is believed to have favorable spin dynamics as a weak spin-orbit interaction in this material should suppress spin relaxation mechanisms involving spin-orbit coupling such as Dyakonov-Perel mechanism [7] or Elliot-Yaffet mechanisms [8,9]. Moreover, since both zinc and oxygen atoms have a high natural abundance (around 95\% and 99\%, respectively) of nuclear-spin free isotopes, spin relaxation via electron-nuclear hyperfine coupling should also be strongly suppressed.

Experimentally, studies of spin dynamics in $\mathrm{ZnO}$ have so far been carried out for effective-mass electrons and holes that were either free or localized at shallow donors. Relatively long spin-coherence times, $1-25 \mathrm{~ns}$ at $4 \mathrm{~K}$ and $0.19 \mathrm{~ns}$ at RT, were measured for free and donor-bound electrons in bulk and epitaxial $\mathrm{ZnO}$, from time-resolved Faraday rotation [10] and spin noise spectroscopy [11]. An even longer spin coherence time of $25 \mathrm{~ns}$ was reported by Liu et al. [12] for electrons in $n$-type colloidal quantum dots based on electron paramagnetic resonance spectroscopy. Effective-mass holes have been found to exhibit significantly faster spin relaxation, i.e. $100 \mathrm{ps}$ at $4 \mathrm{~K}$ in bulk $\mathrm{ZnO}$ and $350 \mathrm{ps}$ in strained epilayers, based on time-resolved optical orientation [13] and magneto-optical $[14,15]$ measurements. These studies were, however, limited to holes in the neutral donor bound exciton (BX) complexes due to difficulties in fabrication of a stable $p$-type material of reasonable quality.

As to spin dynamics of electron-hole pairs in a form of excitons that is relevant, e.g. to optoelectronic applications, the information available so far is very limited. It has solely been restricted to free excitons (FXs) where extremely fast
$(<10$ ps at $4 \mathrm{~K})$ spin relaxation was concluded based on the lack of FX polarization in optical orientation measurements [13]. Recently, we have shown [16] that excitons in ZnO may also be bound to isoelectronic centers forming isoelectronic bound excitons (IBXs). These isoelectronic centers have a hole attractive potential which leads to tight binding of the hole within the IBX complex and to partial quenching of its orbital angular momentum. Implications of such an electronic structure on exciton spin dynamics are currently not known and will be addressed in this paper by employing time-resolved optical spin orientation spectroscopy.

\section{SAMPLES AND METHODS}

Bulk single crystals investigated in this study include undoped $c$-plane $\mathrm{ZnO}$ from Cermet Inc. and $c$-plane $\mathrm{ZnO}$ implanted with hydrogen from EaglePicher Technologies. Optical measurements were performed within a temperature range of 5-30 $\mathrm{K}$ inside a cryofree cryostat. A wavelength tunable, pulsed Ti:Sapphire laser with a repetition rate of $76 \mathrm{MHz}$ was used as an excitation source. The temporal and spectral widths of the excitation pulses were 2 ps and $1.3 \mathrm{meV}$, respectively. Transient photoluminescence (PL) was detected by a streak camera system combined with a $0.5-\mathrm{m}$ single grating monochromator. Circular polarization of the excitation beam in optical spin orientation experiments was produced by a $\lambda / 4$ plate in combination with a linear polarizer. The same selection of polarization optics was also used to determine a circular polarization degree of PL. The latter is defined in percentage by $P_{c}=100\left(I^{+}-I^{-}\right) /\left(I^{+}+I^{-}\right)$, where $I^{+}$and $I^{-}$denote the intensities of $\sigma^{+}$and $\sigma^{-}$polarized PL, respectively. Both excitation and detection directions coincided with the direction of the $c$ axis in $\mathrm{ZnO}$.

\section{EXPERIMENTAL RESULTS AND DISCUSSION}

\section{A. Optical orientation}

Typical PL spectra measured at $5 \mathrm{~K}$ from the Cermet (the solid curve) and EaglePicher (the dashed curve) $\mathrm{ZnO}$ are shown in Fig. 1. In both materials, the low temperature PL spectra are dominated by sharp BX transitions. The majority of these lines originate from $\mathrm{BX}$ bound to either neutral $\left(D^{0} X\right)$ or ionized $\left(D^{+} X\right)$ donors. The former include PL lines related 


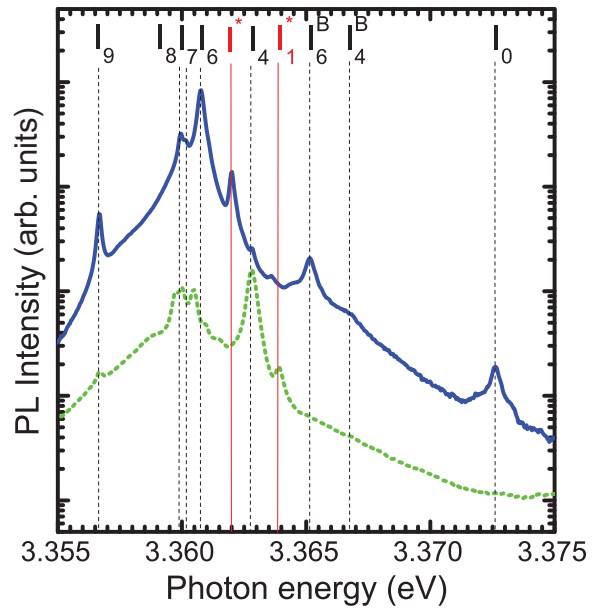

FIG. 1. (Color online) Photoluminescence spectra measured at $5 \mathrm{~K}$ from the Cermet (upper curve) and EaglePicher (lower curve) $\mathrm{ZnO}$. Donor bound excitons are marked by the dashed vertical lines, whereas the $I^{*}$ and $I_{1}^{*}$ isoelectronic BXs are marked by the solid vertical lines.

to $D^{0} X$ involving a hole from either A- or B-valence subbands and are labeled as $I_{4}, I_{6}-I_{9}$, or $I_{4}{ }^{\mathrm{B}}$ and $I_{6}{ }^{\mathrm{B}}$, respectively [17-19]. $D^{+} X$ gives rise to a weak transition labeled as $I_{0}$, following the notations from the literature [17,18]. In addition to the aforementioned donor bound excitons, the spectra contain PL lines labeled as $I^{*}$ and $I_{1}^{*}$ that are most pronounced for the Cermet and EaglePicher $\mathrm{ZnO}$, respectively. Our previous magneto-optical measurements have proved that these transitions belong to a new class of excitons in $\mathrm{ZnO}$, i.e. excitons bound at isoelectronic centers with a hole-attractive local potential [16].

In order to understand spin properties of this class of excitons, optical orientation experiments were performed. In such experiments, a chosen spin orientation of excitons may be generated by the corresponding circularly polarized excitation light. In wurtzite semiconductors, such as $\mathrm{ZnO}$, combined effects of crystal field and spin-orbit interactions lead to a splitting of the valence band (VB) states into the so-called $\mathrm{A}, \mathrm{B}$, and $\mathrm{C}$ subbands. In a measurement configuration where light propagates along the $c$ axis of the crystals (i.e. $k \| c$ ), the selection rules allow optical transitions between the conduction band states of the $\Gamma_{7}$ symmetry and the $\mathrm{A}\left(\Gamma_{7}\right)$ and B $\left(\Gamma_{9}\right)$ VB states [20]. Since, the A- and B-valence states are constructed from the VB states with the total (spin + orbital) angular momentum projection $J_{h, z}= \pm 1 / 2$ and $\pm 3 / 2$, respectively, absorption of the circularly polarized light due to the $\mathrm{A} \rightarrow \mathrm{CB}$ and $\mathrm{B} \rightarrow \mathrm{CB}$ transitions will generate opposite spin orientations of the $\mathrm{CB}$ electrons. The same also applies to the corresponding FX. Therefore, in order to optically polarize the exciton spin, the excitation energy of the circular-polarized (e.g. $\sigma^{-}$) light in our experiments was tuned below the $\mathrm{B}$-free exciton $\left(\mathrm{FX}_{\mathrm{B}}\right)$. The results from the optical orientation measurements of $I^{*}$ and $I_{1}^{*}$ are summarized in Figs. 2(a) and 2(b), respectively. It was found that such excitation indeed causes circular polarization of the time-integrated $I^{*}$ and $I_{1}^{*}$ emissions and that the generated $P_{c}$ critically depends on the excitation energy $\left(h v_{\text {exc }}\right)$. This can be seen from the right part
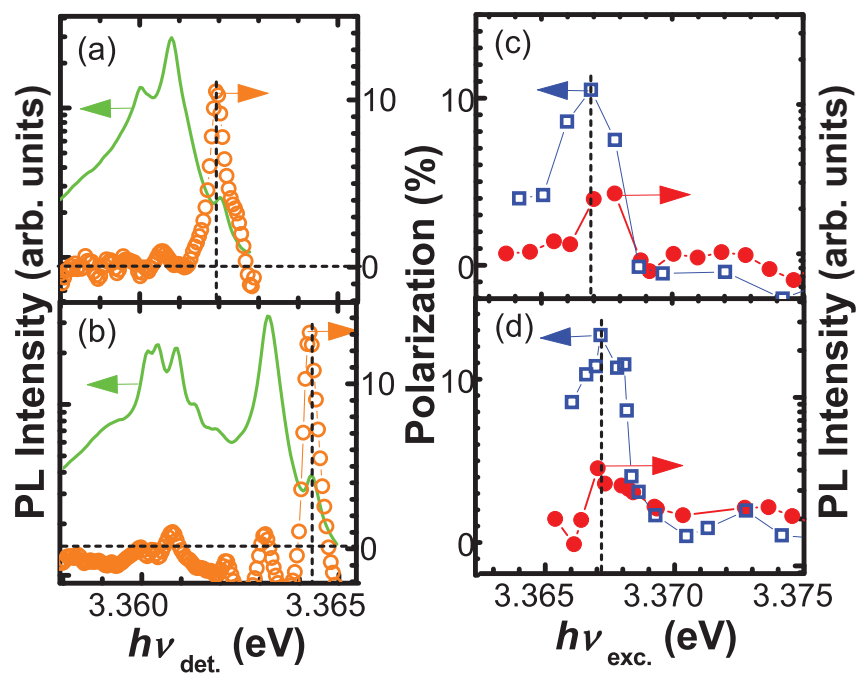

(e)

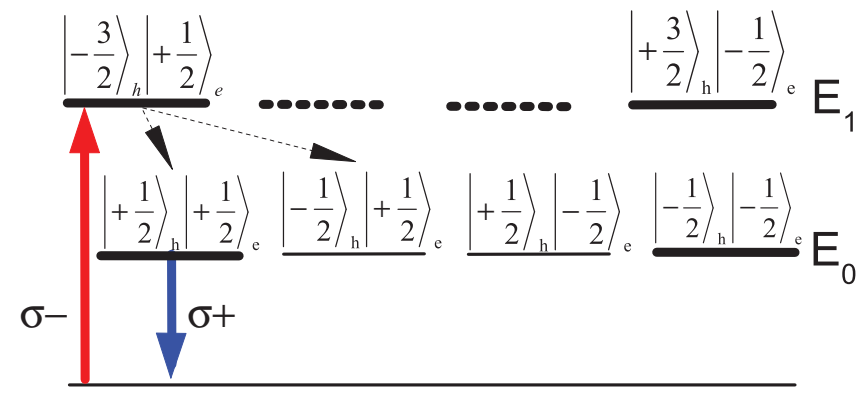

FIG. 2. (Color online) Spectral dependences of the PL circular polarization $P_{c}$ (open circles) measured as a function of emission photon energy from the (a) Cermet and (b) EaglePicher $\mathrm{ZnO}$. The excitation photon energy $h v_{\text {exc }}$ is fixed at $E_{1}$, marked by the vertical dashed line in (c) and (d), at which the maximum value of $P_{c}$ is obtained for each IBX. Also shown are respective time-integrated PL spectra (solid curves) measured under linear laser excitation with the same $h v_{\text {exc }}$. (c) and (d) show the spectral dependences of the PL circular polarization $P_{c}$ (the open squares) measured as a function of excitation photon energy from the Cermet and EaglePicher $\mathrm{ZnO}$, respectively, by detecting at the peak positions of the $I^{*}$ and $I_{1}{ }^{*}$ exciton, i.e. $E_{0}$ of the IBX as marked by the vertical dashed line in (a) and (b). The corresponding excitation spectra of the PL intensity are shown by the filled circles. (e) Schematic energy structure of an isoelectronic BX. The energy structure of the exciton ground state $E_{0}$ is taken from Ref. [16]. $E_{1}$ denotes the exciton excited state. The solid arrows represent optical absorption and emission transitions which lead to the detected $\sigma^{+}$polarization under optical orientation with the $\sigma^{-}$-polarized excitation light. The dotted arrows denote energy relaxation processes that preserve electron spin orientation within the IBX.

of Fig. 2 where the measured $P_{c}$ (the open squares) is plotted as a function of $h v_{\text {exc }}$. It is obvious that $P_{c}$ is resonantly enhanced and reaches the maximum value of $+10(+13) \%$ for $I^{*}\left(I_{1}^{*}\right)$ when $h v_{\text {exc }}$ is tuned at about $4 \mathrm{meV}$ above the emission energy, i.e. at 3.3668 (3.3673) eV, but becomes negligible for $h v_{\mathrm{exc}}$ exceeding $3.369 \mathrm{eV}$. Figures 2(a) and 2(b) show $P_{c}$ (the open circles) as a function of detection energy $\left(h v_{\text {det }}\right)$ measured from the Cermet and EaglePicher ZnO. Here, the PL was excited 
at the energy that provides the maximum polarization in each sample, i.e. at $h v_{\mathrm{exc}}=3.3668$ and $3.3673 \mathrm{eV}$, respectively. For easy reference, also shown are PL spectra of these samples (the solid curves). Among all BX, a sizable PL polarization degree under such excitation conditions is only generated for the $I^{*}$ and $I_{1}^{*}$ excitons which implies that the observed resonance in the $P_{c}\left(h v_{\text {exc }}\right)$ dependences should be related to the intrinsic energy structure of these IBX. This assumption is further confirmed by PL excitation (PLE) measurements performed on $I^{*}$ and $I_{1}^{*}$. The corresponding PLE spectra are shown by solid dots in Figs. 2(c) and 2(d), respectively, and exhibit resonant enhancement at the same excitation energies as those required to generate $P_{c}$.

Let us now discuss the origin of the observed polarization. From Figs. 2(c) and 2(d), it is reasonable to suggest that the optical orientation of $I^{*}$ (or $I_{1}^{*}$ ) by circularly polarized light is achieved under resonant excitation at a higher lying excited state (labeled as $E_{1}$ ) of this exciton located at around $4 \mathrm{meV}$ above the ground state (labeled as $E_{0}$ ), as shown schematically in Fig. 2(e). The PL polarization degree is positive, i.e. the emitted light has an opposite helicity to the excitation light. This implies that the projection of the angular momentum of the IBX complex differs between its excited and ground states. According to our previous magneto-optical studies [16], the ground state of the $I^{*}$ and $I_{1}^{*}$ excitons is formed from electron and hole states with an effective spin $1 / 2$. The electron wave function is $s$-like, i.e. $\left| \pm \frac{1}{2}\right\rangle_{e}$, whereas the hole component of the wave function is constructed as $\left|+\frac{1}{2}\right\rangle_{h}=-\gamma|+1, \Downarrow\rangle-$ $\delta \mid 0$, $\rangle\rangle$ and $\left|-\frac{1}{2}\right\rangle_{h}=\gamma|-1, \Uparrow\rangle+\delta|0, \Downarrow\rangle$. Here, $\left|+\frac{1}{2}\right\rangle_{e}\left(\left|-\frac{1}{2}\right\rangle_{e}\right)$ denotes the spin-up (spin-down) electron state, i.e. $\left|+\frac{1}{2}\right\rangle_{e}=$ $|\uparrow\rangle\left(\left|-\frac{1}{2}\right\rangle_{e}=|\downarrow\rangle\right)$, and $\left|m_{l}, \Uparrow\right\rangle\left(\left|m_{l}, \Downarrow\right\rangle\right)$ denotes the hole state constructed from the orbital state $\left|m_{l}\right\rangle$ and spin-up (spin-down) spin state. The parameters $\gamma$ and $\delta$ describe mixing of the $\left|m_{l}= \pm 1\right\rangle$ and $\left|m_{l}=0\right\rangle$ states of the hole, with $\gamma^{2}+\delta^{2}=1$ and $\gamma^{2} \gg \delta^{2}$. This is to a certain extent similar to the A-VB hole states in $\mathrm{ZnO}$, though the contribution from the $\left|m_{l}=0\right\rangle$ state is significantly higher for the holes tightly bound at the isoelectronic $I^{*}$ and $I_{1}^{*}$ centers, likely due to combined effects of the spin-orbit interaction and local crystal field. The ground exciton state is fourfold degenerate and contains two bright states, i.e $\left|+\frac{1}{2}\right\rangle_{h}\left|+\frac{1}{2}\right\rangle_{e}$ and $\left|-\frac{1}{2}\right\rangle_{h}\left|-\frac{1}{2}\right\rangle_{e}$ that emit light in the $\sigma^{+}$and $\sigma^{-}$polarization, respectively, and two dark states that are spin forbidden in optical transitions-see Fig. 2(e). The excited state of these excitons should then contain either $p$-like electron or $|+1, \Uparrow\rangle$ and $|-1, \Downarrow\rangle$ hole states, to account for the observed counter-polarization of the $I^{*}$ and $I_{1}^{*}$ emissions in the optical orientation experiments-see Figs. 2(a)-2(d). Since the electron within the BX complex is effective mass like [15], the former is highly unlikely. Indeed, the energy difference between the $1 s$ and $2 p$ states of the effective mass donor in $\mathrm{ZnO}$ is around $30-40 \mathrm{meV}$ [17], i.e. by far too large to account for the 4.7 (3.2) meV energy separation between $E_{0}$ and $E_{1}$ of the $I^{*}\left(I_{1}^{*}\right)$ center. We, therefore, suggest that the $E_{1}$ state is constructed from the $\left| \pm \frac{1}{2}\right\rangle_{e}$ electron state and $\left| \pm \frac{3}{2}\right\rangle_{h}$ hole state (i.e. $\mid+1$, $\left.\rangle\right\rangle$ and $|-1, \downarrow\rangle$, possibly with some admixture of other hole states). This seems to indicate that the hole in the $E_{1}$ state couples to the B-VB states, which is in a way consistent with the energy separation between $E_{0}$ and $E_{1}$ that is very close to the A-B splitting. Resonant excitation of $E_{1}$ by the $\sigma^{-}$-polarized light will generate population of the $\left|-\frac{3}{2}\right\rangle_{h}\left|+\frac{1}{2}\right\rangle_{e}$ state. If the generated electron spin orientation is preserved within the $E_{1}$ lifetime and during subsequent energy relaxation to $E_{0}$, this will in turn lead to the population of the bright $\left|+\frac{1}{2}\right\rangle_{h}\left|+\frac{1}{2}\right\rangle_{e}$ state of $E_{0}$ and thus positive $P_{c}$, as shown schematically in Fig. 2(e).

We also note that absorption of the circularly polarized light by $\mathrm{FX}_{\mathrm{A}}$ does not generate spin polarization of either $I^{*}$ or $I_{1}^{*}$ excitons. This can be attributed to combined effects of fast spin relaxation of the free exciton [13] and spin loss during its capture by the isoelectronic centers.

\section{B. Spin dynamics}

The measured $P_{c}$ values of time-integrated $I^{*}$ and $I_{1}^{*}$ emissions are relatively low, which suggest either incomplete electron spin generation at $E_{1}$ after the excitation process or spin loss at $E_{0}$ caused by spin relaxation. In order to achieve a better understanding of the IBX spin dynamics, we have performed time-resolved PL measurements under circularly $\left(\sigma^{-}\right)$polarized pulsed excitation which was tuned in resonance with the $E_{1}$ excited state. Representative decays of the PL polarization measured after the laser pulses at three different temperatures are shown in Figs. 3(a)-3(c) (the open circles), taking as an example the $I^{*}$ emission. Also shown are decays of the total PL intensity $\left(I^{+}+I^{-}\right)$, represented by the solid lines. The decays of the PL polarization clearly contain several components which indicate involvement of different processes of optical depolarization. The first process, which is faster than the instrumental response of $10 \mathrm{ps}$, readily reduces the degree of PL polarization down to about $11 \%$ (at $4 \mathrm{~K}$ ) for $I^{*}$ and $13 \%$ for $I_{1}^{*}$. It is followed by a relatively fast $P_{c}$ decrease within the first 400 ps after which the degree of PL polarization remains practically constant

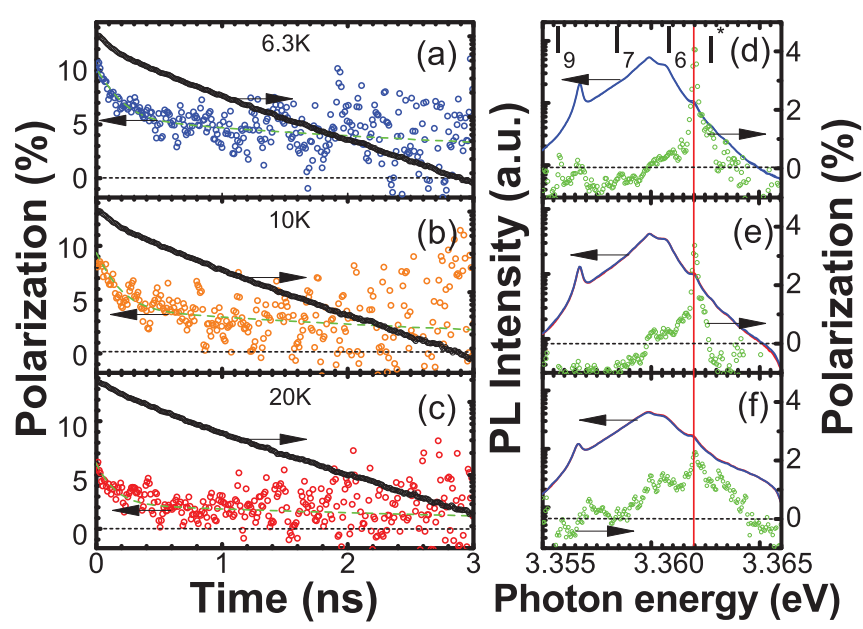

FIG. 3. (Color online) (a)-(c) Measured temporal decays of the total PL intensity (solid lines) and circular polarization (open circles) for the $I^{*}$ emission at the specified temperatures. The dashed lines are just a guide to the eye to illustrate the $P_{c}$ decays. (d)-(f) Photoluminescence spectra (solid lines) and $P_{c}\left(h v_{\mathrm{det}}\right)$ dependences that were time-integrated within the window of $2-5 \mathrm{~ns}$ after the end of the excitation pulse. 
with further time delay $\Delta t_{d}$ after the excitation pulse. The same temporal behavior of the PL polarization was observed for all measurement temperatures between 6.3 and $30 \mathrm{~K}$, though the initial values of $P_{c}$ continuously decreased at higher temperatures. Since the experimental data become rather noisy when $\Delta t_{d}$ exceeds $2 \mathrm{~ns}$, the existence of $P_{c}$ in this temporal range was further confirmed by measuring $P_{c}$ as a function of emission energy that were integrated within the time interval of $\Delta t_{d}=2-5 \mathrm{~ns}-$ see Figs. 3(d)-3(f). Undoubtedly, the $I^{*}$ (and also $I_{1}^{*}$ ) emission remains circularly polarized within this time window, and the measured $P_{c}$ corresponds to the values reached after the initial $P_{c}$ decays depicted in Figs. 3(a)-3(c). From Fig. 3, it is also noticeable that the fast temporal decay of $P_{c}$ coincides with the fast decay of the total PL intensity. The latter is found to be biexponential and to contain fast and slow decay components with the corresponding decay times of 0.18 and $0.75 \mathrm{~ns}$, respectively. Though these time constants are found to be temperature independent within the range of 4-30 K, the contribution of the fast PL decay component decreases with increasing temperature.

The results of the performed transient PL and $P_{c}$ measurements can be explained within two models which differ in interpretation of the biexponential character of the PL decay. The first model assumes that the fast component of this decay reflects spin dynamics of IBX and is caused by exciton transfer between the bright and dark states. Within the second model, on the other hand, two PL decay components are considered to simply stem from excitons recombining in two spatial regions with distinctly different carrier lifetimes such as surface and bulk areas of $\mathrm{ZnO}$. Let us now discuss these models in more detail.

\section{Model I}

The fourfold degenerate ground state $E_{0}$ of the $I^{*}$ and $I_{1}{ }^{*}$ excitons contains two bright and two dark states. The resonant optical excitation of the excitons at $E_{1}$ will populate the bright states of $E_{1}$ with the specific angular momentum that is allowed by the electric-dipole selection rule. After these $E_{1}$ excitons are optically generated, they will undergo population transfer between the sublevels of $E_{1}$ and, more significantly, energy relaxation down to the exciton ground state $E_{0}$. As it is reasonable to assume that the energy relaxation processes that conserve electron spin angular momentum should be more probable than the spin-flipping ones, it is expected that two of the four sublevels of $E_{0}$, namely the $\sigma^{+}$-active $\left|+\frac{1}{2}\right\rangle_{h}\left|+\frac{1}{2}\right\rangle_{e}$ and the dark $\left|-\frac{1}{2}\right\rangle_{h}\left|+\frac{1}{2}\right\rangle_{e}$, are more favorably fed from $E_{1}$ under the $\sigma^{-}$excitation [see Fig. 2(e)]. If the same applies to the hole spin during energy relaxation, the bright $\left|+\frac{1}{2}\right\rangle_{h}\left|+\frac{1}{2}\right\rangle_{e}$ is expected to be more populated than the dark $\left|-\frac{1}{2}\right\rangle_{h}\left|+\frac{1}{2}\right\rangle_{e}$. After the excitation pulses, populations of all four states within $E_{0}$ will start to equalize due to electron, hole, and exciton spin relaxation with characteristic time constants of $\tau_{e}^{s}, \tau_{h}^{s}$, and $\tau_{e h}^{s}$, respectively-see Fig. 4(a). This could lead to the appearance of the biexponential PL decays, provided that spin relaxation time is shorter than the exciton lifetime. For a quantitative description of the spin and recombination dynamics at the exciton ground state, we resort to the following set of rate equations:

\section{(a) Model I}
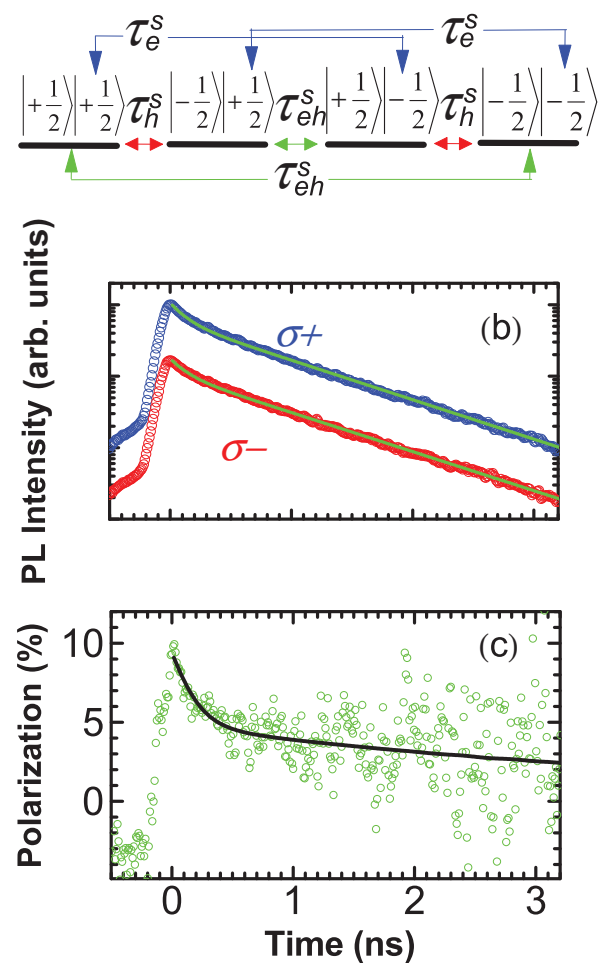

FIG. 4. (Color online) (a) Schematic illustration of the spin-flip transitions in the exciton ground state $E_{0}$, which were considered in the rate equation analysis of the spin and recombination dynamics of the IBX. (b) Decays of the $\sigma^{+}$- and $\sigma^{-}$-polarized $I^{*}$ emission and (c) the corresponding PL circular polarization measured at $6.3 \mathrm{~K}$ under the $\sigma^{-}$-polarized resonant excitation at the $E_{1}$ state. The open circles represent experimental data, whereas the solid lines are fitting curves based on model I, obtained using Eqs. (1)-(4) with the fitting parameters given in the text. The PL decays are vertically offset, for clarity.

$$
\begin{aligned}
\frac{d n_{++}}{d t}= & -\frac{n_{++}}{\tau_{r}}+\left(\frac{n_{-+}-n_{++}}{\tau_{h}^{s}}\right)+\left(\frac{n_{+-}-n_{++}}{\tau_{e}^{s}}\right) \\
& +\left(\frac{n_{--}-n_{++}}{\tau_{e h}^{s}}\right), \\
\frac{d n_{-+}}{d t}= & -\frac{n_{-+}}{\tau_{r}^{\prime}}+\left(\frac{n_{++}-n_{-+}}{\tau_{h}^{s}}\right)+\left(\frac{n_{--}-n_{-+}}{\tau_{e}^{s}}\right) \\
& +\left(\frac{n_{+-}-n_{-+}}{\tau_{e h}^{s}}\right), \\
\frac{d n_{+-}}{d t}= & -\frac{n_{+-}}{\tau_{r}^{\prime}}+\left(\frac{n_{--}-n_{+-}}{\tau_{h}^{s}}\right)+\left(\frac{n_{++}-n_{+-}}{\tau_{e}^{s}}\right) \\
& +\left(\frac{n_{-+}-n_{+-}}{\tau_{e h}^{s}}\right), \\
\frac{d n_{--}}{d t}= & -\frac{n_{--}+\left(\frac{n_{+-}-n_{--}}{\tau_{r}^{s}}\right)+\left(\frac{n_{-+}-n_{--}}{\tau_{e}^{s}}\right)}{}+\left(\frac{n_{++}-n_{--}}{\tau_{e h}^{s}}\right) .
\end{aligned}
$$


Here, $n_{++}, n_{-+}, n_{+-}$, and $n_{--}$are exciton populations at the $\left|+\frac{1}{2}\right\rangle_{h}\left|+\frac{1}{2}\right\rangle_{e},\left|-\frac{1}{2}\right\rangle_{h}\left|+\frac{1}{2}\right\rangle_{e},\left|+\frac{1}{2}\right\rangle_{h}\left|-\frac{1}{2}\right\rangle_{e}$, and $\left|-\frac{1}{2}\right\rangle_{h}\left|-\frac{1}{2}\right\rangle_{e}$ sublevels of $E_{0}$, respectively. $\tau_{r}\left(\tau_{r}^{\prime}\right)$ represents the lifetime of the bright (dark) states. In the simulations, $\tau_{r}$ was set to $0.7 \mathrm{~ns}$, i.e. to the value comparable with the decay time of donor bound excitons. It was also assumed that the hole relaxation time is shorter than that for the electron, as commonly observed in semiconductor materials. The best fit to the measured decays of the $I^{*}$ intensity $I_{\mathrm{PL}}(t)$ and its polarization $P_{c}(t)$ yields $\tau_{r}^{\prime}=0.9 \pm 0.05 \mathrm{~ns}, \tau_{h}^{s}=0.4 \pm 0.05 \mathrm{~ns}, \tau_{e}^{s}=15 \div 25 \mathrm{~ns}$, and $\tau_{e h}^{s}=15 \div 30 \mathrm{~ns}$. The low accuracy in determining the $\tau_{e}^{s}$ and $\tau_{e h}^{s}$ values is due to the limited time range available for the analysis, which is restricted by the exciton lifetime. The simulated transients of the $\sigma^{+}$- and $\sigma^{-}$-polarized PL components, as well as of $P_{c}(t)$ are shown by the solid lines in Figs. 4(b) and 4(c), respectively, together with the experimental data (the open circles) obtained under the $\sigma^{-}$ excitation.

A comparable value of $\tau_{h}^{s}$ with that of the fast $P_{c}$ decay indicates that the initial decrease of $P_{c}$ is caused by hole spin relaxation, which transfers exciton population from the initially more populated $\left|+\frac{1}{2}\right\rangle_{h}\left|+\frac{1}{2}\right\rangle_{e}$ state to the $\left|-\frac{1}{2}\right\rangle_{h}\left|+\frac{1}{2}\right\rangle_{e}$ state until they reach a dynamical balance between them. In contrast, the processes involving electron and exciton spin flips, see Fig. 4(a), are much longer than the exciton lifetime. This gives rise to a nearly constant value of $P_{c}$ for time delays exceeding $0.6 \mathrm{~ns}$.

Model I can also explain the results from the temperaturedependent measurements shown in Fig. 3. The decays of both PL intensity and its circular polarization can be reproduced assuming temperature-independent values of all time constants. The decreasing contribution of the fast decay components at higher temperatures can be attributed to a decrease in the population difference between the bright and dark states immediately after the end of the excitation pulse, i.e. at $\Delta t_{d}=$ 0 . This in turn means acceleration of the spin relaxation during the feeding process, i.e. either at the excited $E_{1}$ state or/and during the energy relaxation from $E_{1}$ to $E_{0}$. This accelerated spin loss can also explain the observed decrease in the initial $P_{c}$ value (i.e. at $\Delta t_{d}=0$ ) with increasing measurement temperature, as this process should equalize populations of two bright states of $E_{0}$ either via subsequent electron and hole spin flips or via direct exciton spin-flip.

\section{Model II}

Our previous transient study of $D^{0} X$ [21] has shown that these transitions in $\mathrm{ZnO}$ often exhibit a biexponential decay which reflects contributions of exciton emissions from two spatial regions with distinctly different exciton lifetimes, i.e. close to the surface (short lifetime) and in bulk (long lifetime). In model II, it is assumed that the same could be true for the isoelectonic $I^{*}$ and $I_{1}{ }^{*}$ excitons, such that the observed PL decay is a superposition of the two contributions, i.e.

$$
I_{\mathrm{PL}}=A_{f} \exp \left(-\frac{t}{\tau_{f}}\right)+A_{s} \exp \left(-\frac{t}{\tau_{s}}\right) .
$$

Here, $A_{f}\left(A_{s}\right)$ denotes the amplitude of the surface-related fast (bulk-related slow) component with the time constant of $\tau_{f}$ $\left(\tau_{s}\right)$. In the case of $D^{0} X$, the ratio between $A_{f}$ and $A_{s}$ depends (a) Model II

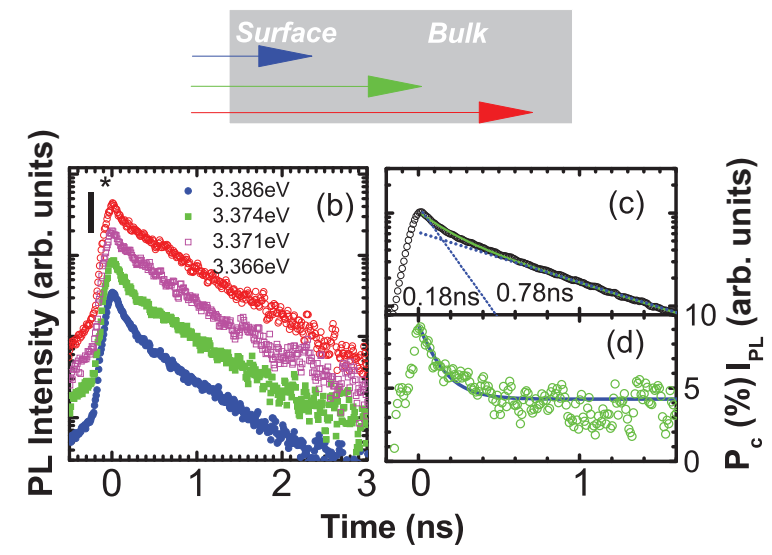

FIG. 5. (Color online) (a) Schematic illustration of relative contributions of the surface-related and bulk emissions with respective to the excitation energy and thus the penetration depth of the excitation light. (b) Decays of the total intensity of the $I^{*}$ emission measured at $6.3 \mathrm{~K}$ using different excitation energies of linearly polarized light. (c) Decay of the total intensity of the $I^{*}$ emission measured at $6.3 \mathrm{~K}$ under excitation with linearly polarized light tuned in resonance with the $E_{1}$ state (the open circles). The dotted lines are fitting curves using Eq. (5). (d) Decay of the $I^{*}$ circular polarization measured at $6.3 \mathrm{~K}$ under the $\sigma^{-}$-polarized resonant excitation at the $E_{1}$ state (the open circles). The solid line is a fitting curve obtained based on model II.

on the excitation energy $\left(h v_{\mathrm{exc}}\right)$ due to variations in penetration depth of the laser light [21]—-see Fig. 5(a). The contribution of the surface-related recombination continuously decreases when the excitation energy is tuned below $\mathrm{FX}_{\mathrm{A}}$ and is moved farther away from it. To verify whether the same behavior is also observed for the isoelectronic bound excitons, PL decays of $I^{*}$ were measured under different $h v_{\text {exc }}$. The corresponding results are shown in Fig. 5(b) and show the same trend of decreasing $A_{f}$ with increasing $h v_{\text {exc }}$ as was observed for $D^{0} X$. This finding, therefore, is consistent with the suggestion that the fast component of the PL transient reflects the $I^{*}$ lifetime in the near-surface region. (We cannot rule out model I based on this finding, however, as a change in $h v_{\text {exc }}$ may also result in different populations of the dark and bright states of $E_{0}$ and, therefore, affect the relative contribution of the fast PL decay.) Using model II, the measured decays of the PL intensity and polarization can be reproduced assuming that $\tau_{f}=0.18 \div 0.02 \mathrm{~ns}, \tau_{s}=0.75 \div 0.03 \mathrm{~ns}$, and that $P_{c}$ remains constant within the PL lifetime, as demonstrated in Figs. 5(c) and 5(d) by a comparison between the simulation (the solid lines) and experimental data (the open circles) measured at $6.3 \mathrm{~K}$ for the $I^{*}$ emission. The $P_{c}$ values of the fast and slow component were kept constant in time and equal to 20 and $4 \%$, respectively. We note that, in order to reproduce the experimental data, the $P_{c}$ value needs to be significantly higher for the fast PL component. Though such a large difference in the $P_{c}$ values is somewhat surprising, it could in principle be attributed to a shorter lifetime of the exciton and consequently a reduced contribution of spin relaxation in the near-surface region as compared with the bulk region. Based on Eqs. (1)-(4), the constant $P_{c}$ in time implies 
that the characteristic times of all spin-flip processes (i.e. for the electron, the hole, and the exciton as a whole) are longer than the exciton lifetimes with the low bound of 10 (15) ns for the near-surface (bulk) regions. An overall decrease of the PL polarization with rising temperature (Fig. 3) can be ascribed to the temperature-induced acceleration of spin relaxation during the feeding process, i.e. similarly to model I. Model II faces difficulties, however, when explaining the suppression of the fast PL decay component at elevated temperatures. Indeed, contributions of the surface-related and bulk $I^{*}\left(I_{1}{ }^{*}\right)$ excitons under resonant excitation via the $E_{1}$ state are expected to be solely determined by the penetration depth of the excitation light, which is temperature independent. This makes this model somewhat less likely.

We, therefore, suggest that, though surface effects may contribute to the spin dynamics, the main physical process responsible for the appearance of the fast decay component in the $I^{*}$ and $I_{1}{ }^{*}$ transients is the spin relaxation from the bright to dark state within the exciton ground state accomplished via a hole spin-flip. The same process also leads to the initial decay of the circular PL polarization in optical orientation experiments.

\section{Origin of spin relaxation}

Let us now briefly discuss spin relaxation processes that govern spin dynamics of the studied isolectronic bound excitons in $\mathrm{ZnO}$. We can first examine the determined time constants of the spin-flip transitions at the exciton ground state. The fastest process is spin relaxation of the bound hole with $\tau_{h}^{s}$ equal to $0.4 \mathrm{~ns}$. Though being relatively short, this value is still longer than the spin-flip time of an effective-mass hole bound within the $D^{0} X$ complex, i.e. 100 ps in bulk $\mathrm{ZnO}$ [13]. The observed slow-down of the hole spin relaxation is probably not surprising as partial quenching of the orbital angular momentum for the tightly bound hole should weaken spin-orbit interaction that usually dominates hole spin dynamics. On the other hand, electron spin relaxation is significantly slower, i.e. $15 \mathrm{~ns}$ or longer at $T<30 \mathrm{~K}$. This is likely a result of localization at the isoelectronic center which deactivates spin relaxation mechanisms related to carrier motion, such as Dyakonov-Perel [7] or Elliot-Yafet processes [8,9]. The determined value of $\tau_{e}^{s}$ is in fact comparable with the spin coherence time of an electron bound to an effective-mass donor in $\mathrm{ZnO}$ ( $25 \mathrm{~ns}$ ) that was concluded to be limited by the hyperfine interaction with nuclear spins of the naturally occurring ${ }^{67} \mathrm{Zn}$ isotope [11]. Moreover, our results show that direct spin relaxation between the bright states via an exciton spin-flip is also a rather slow process characterized by $\tau_{e h}^{s} \geqslant 15 \mathrm{~ns}$. We suggest that this reflects combined effects of a weak $e-h$ exchange interaction for the studied IBX [16] and exciton localization. The latter should diminish importance of the long-range $e-h$ exchange interaction, which has been shown to dominate spin relaxation of free excitons but weakens linearly with decreasing momentum $K$ [22].

From Fig. 3, the initial value of the exciton polarization generated at $E_{0}$ by optical orientation is rather low. As a reminder, this optical orientation is believed to be accomplished via the resonant excitation at the higher lying excited state $\left(E_{1}\right)$ of the isolectronic BX. The low initial value of $P_{c}$ could, therefore, be caused by two factors. First of all, it can reflect poor generation of spin polarization at $E_{1}$ if the states forming $E_{1}$ are spin mixed. Though the exact structure of the $E_{1}$ state could not be determined from the present study, this is very likely as spin mixing (though weak) is known to occur in the exciton ground state. Secondly, it can be a result of fast spin relaxation during the feeding process, i.e. spin loss at $E_{1}$ prior to and during the energy relaxation to $E_{0}$. The contribution of these processes becomes more significant at temperatures exceeding $10 \mathrm{~K}$, since raising temperature causes a decrease in the initial $P_{c}$ values. This thermally accelerated spin relaxation can be caused by, for example, the Orbach-type relaxation process [23].

\section{SUMMARY}

In conclusion, we have employed the time-resolved optical spin orientation technique to study spin dynamics of $I^{*}$ and $I_{1}^{*}$ excitons bound to isoelectronic centers in bulk $\mathrm{ZnO}$ single crystals. We show that spin orientation at the exciton ground state $E_{0}$ can be generated using resonant excitation at a higher lying exciton state located at about $4 \mathrm{meV}$ apart from $E_{0}$. The induced circular PL polarization declines within the first $400 \mathrm{ps}$ after the end of the excitation pulse but remains practically constant with further time delay. The rate equation analysis of the observed spin dynamics at the exciton ground state, which takes into account subsequent hole and electron spin flips between the bright and dark states of $\mathrm{BX}$ and direct exciton spin flips between the bright states of $E_{0}$, allows us to determine the characteristic time constants of these processes as being $\tau_{h}^{s}=0.4 \mathrm{~ns}, \tau_{e}^{s} \geqslant 15 \mathrm{~ns}$, and $\tau_{e h}^{s} \geqslant 15 \mathrm{~ns}$, respectively. These values are substantially longer than spin relaxation times for a free exciton $(<10 \mathrm{ps})$ or an effective-mass hole bound to a donor-bound exciton complex (0.1 ns) that were determined previously in bulk $\mathrm{ZnO}$ [13]. The slowdown of the exciton spin relaxation likely reflects combined effects of exciton localization at the isoelectronic center and a weak exchange interaction of the studied BX. The relatively slow hole spin relaxation in IBX can be attributed to the suppressed spin-orbit coupling for the tightly bound hole. Our findings, therefore, indicate that this class of bound excitons is attractive for applications in spin-functional light emitters.
[1] Ü. Özgür, Y. I. Alivov, C. Liu, A. Teke, M. A. Reshchikov, S. Doğan, V. Avrutin, S.-J. Cho, and H. J. Morkoc, J. Appl. Phys. 98, 041301 (2005).

[2] C. Klingshirn, Phys. Status Solidi B 244, 3027 (2007).
[3] S. J. Pearton, D. P. Norton, M. P. Ivill, A. F. Hebard, J. M. Zavada, W. M. Chen, and I. A. Buyanova, IEEE Trans. Electron Devices 54, 1040 (2007).

[4] T. Dietl, H. Ohno, F. Matsukura, J. Cibert, and D. Ferrand, Science 287, 1019 (2000). 
[5] S. J. Pearton, D. P. Norton, Y. W. Heo, L. C. Tien, M. P. Ivill, Y. Li, B. S. Kang, F. Ren, J. Kelly, and A. F. Hebard, J. Electron. Mater. 35, 862 (2006).

[6] S. Chambers, T. Droubay, C. Wang, K. Rosso, S. Heald, D. Schwartz, K. Kittilstved, and D. Gamelin, Mater. Today $\mathbf{9}$, 28 (2006).

[7] M. I. Dyakonov, Spin Physics in Semiconductors (Springer, New York, 2008).

[8] R. Elliott, Phys. Rev. 96, 266 (1954).

[9] Y. Yafet, in Solid State Physics, edited by F. Seitz, D. Turnbull (Academic, New York, 1963), Vol. 14.

[10] S. Ghosh, V. Sih, W. H. Lau, D. D. Awschalom, S.-Y. Bae, S. Wang, S. Vaidya, and G. Chapline, Appl. Phys. Lett. 86, 232507 (2005).

[11] H. Horn, A. Balocchi, X. Marie, A. Bakin, A. Waag, M. Oestreich, and J. Hubner, Phys. Rev. B, 87, 045312 (2013).

[12] W. K. Liu, K. M. Whitaker, A. L. Smith, K. R. Kittilstved, B. H. Robinson, and D. R. Gamelin, Phys. Rev. Lett. 98, 186804 (2007).

[13] D. Lagarde, A. Balocchi, P. Renucci, H. Carrère, F. Zhao, T. Amand, X. Marie, Z. X. Mei, X. L. Du, and Q. K. Xue, Phys. Rev. B 78, 033203 (2008).
[14] W. M. Chen, I. A. Buyanova, A. Murayama, T. Furuta, Y. Oka, D. P. Norton, S. J. Pearton, A. Osinsky, and J. W. Dong, Appl. Phys. Lett. 92, 092103 (2008).

[15] I. A. Buyanova, A. Murayama, T. Furuta, Y. Oka, D. P. Norton, S. J. Pearton, A. Osinsky, J. W. Dong, C. W. Tu, and W. M. Chen, J. Supercond. Novel Magn. 23, 161 (2010).

[16] S. L. Chen, W. M. Chen, and I. A. Buyanova, Phys. Rev. B 86, 235205 (2012).

[17] B. K. Meyer, J. Sann, S. Eisermann, S. Lautenschlaeger, M. R. Wagner, M. Kaiser, G. Callsen, J. S. Reparaz, and A. Hoffmann, Phys. Rev. B 82, 115207 (2010).

[18] B. K. Meyer, H. Alves, D. M. Hofmann, W. Kriegseis, D. Forster, F. Bertram, J. Christen, A. Hoffmann, M. Straßburg, M. Dworzak, U. Haboeck, and A. V. Rodina, Phys. Status Solidi B 241, 231 (2004).

[19] S. L. Chen, W. M. Chen, and I. A. Buyanova, Appl. Phys. Lett. 99, 091909 (2011).

[20] D. G. Thomas, J. Phys. Chem Solids 15, 86 (1960).

[21] S. L. Chen, W. M. Chen, and I. A. Buyanova, Appl. Phys. Lett. 102, 121103 (2013).

[22] M. Z. Maialle, E. A. de Andrade e Silva, and L. J. Sham, Phys. Rev. B 47, 15776 (1993).

[23] C. B. P. Finn, R. Orbach and W. P. Wolf, Proc. Phys. Soc., (London) 77, 261 (1961). 